\title{
¿Incide la inimputabilidad en la atribución del dolo? Eventuales repercusiones en las medidas de seguridad ${ }^{*}$
}

\author{
Osvaldo Artaza V. \\ Raúl Carnevali R.** \\ Recibido: 20 de marzo de 2018 • Aprobado: 06 de junio de 2018 \\ https://doi.org/10.22395/ojum.v17n34a1
}

\begin{abstract}
RESUMEN
El presente artículo tiene por objeto analizar si frente a los supuestos en que el acusado presenta un trastorno psíquico que le impida conocer la naturaleza de su comportamiento, tal condición debiera ser considerada exclusivamente en sede de culpabilidad o si, previamente, la misma debiera ser tenida en cuenta a la hora de definir si al sujeto se le puede o no atribuir un comportamiento doloso, por ende, en sede de imputación subjetiva de la conducta. Para tales efectos, se aborda principalmente el problema de las condiciones que deben ser consideradas relevantes a la hora de atribuir el dolo y el papel que debieran jugar las condiciones propias de la individualidad de un sujeto. Por último, se analiza si la consideración de tales condiciones en sede de imputación subjetiva debiera o no traer alguna consecuencia a la hora de determinar si al inimputable se le puede imponer una medida de seguridad.
\end{abstract}

Palabras clave: inimputabilidad; atribución de dolo; error de tipo psíquicamente condicionado; medidas de seguridad.

\footnotetext{
El presente estudio corresponde, en lo medular, a un informe en derecho encargado a los autores por la Defensoría Penal Pública de Chile durante el año 2017, en atención a un proyecto de especialización de defensores penales públicos a cargo de la defensa de inimputables por enajenación mental. Se agradece la valiosa ayuda prestada por los ayudantes de la Facultad de Derecho y Ciencias Sociales de la Universidad de Talca, Mario Orellana Zenteno y Ricardo Mendoza Tapia.

* Licenciado en Ciencias Jurídicas y Sociales, Universidad de Chile, Santiago, Chile; magíster en Derecho Penal y Procesal Penal, Universidad Alberto Hurtado, Santiago, Chile; doctor en Derecho y Ciencias Políticas, Universidad de Barcelona, Barcelona, España. Profesor asistente de Derecho Penal, Universidad de Talca, Talca, Chile. Correo electrónico: oartaza@utalca.cl.

*** Licenciado en Ciencias Jurídicas y Sociales, Universidad Gabriela Mistral, Santiago, Chile; doctor en Derecho, Universidad de Navarra, Pamplona, España. Profesor titular de Derecho Penal, Universidad de Talca, Talca, Chile. Correo electrónico: rcarnevali@utalca.cl.
} 


\title{
Does the un-imputability have any bearing on the attribution of malice? Possible repercussions on security measures
}

\begin{abstract}
The purpose of this paper is to analyze whether, in cases where the accused has a mental disorder that prevents them from knowing the nature of their conduct, such condition should be considered exclusively in the seat of guilt, or whether it should be taken into account when defining whether the subject may or may not be attributed to fraudulent conduct, thus in the seat of subjective imputation of the conduct. For such effects, the problem of the conditions that must be considered relevant at the time of attributing the fraud and the role that the conditions of the individuality of a subject should play is mainly addressed. Finally, it is analyzed whether or not the consideration of such conditions in the case of subjective imputation should bring any consequence when determining whether a security measure can be imposed on the un-attributable party.
\end{abstract}

Keywords: un-imputability; attribution of malice; error of a psychically conditioned type; security measures.

\section{A inputabilidade incide na atribuição do dolo? Eventuais repercusões nas medidas de seguranças}

\section{RESUMO}

O presente artigo tem por objetivo analisar se diante da possibilidade de que o acusado apresente um transtorno psíquico que o impeça de conhecer a natureza de seu comportamento, tal condição deveria ser considerada exclusivamente em sede de culpabilidade ou se, previamente, a condição deveria ser levada em consideração na hora de definir a atribuição ou não de um comportamento doloso, portanto, em sede de imputação subjetiva da conduta. Para tais efeitos, aborda-se principalmente o problema das condições que devem ser consideradas relevantes na hora de atribuir o dolo e o papel que as condições próprias da individual de um sujeito deveria assumir. Por último, analisa-se se a consideração de tais condições em sede de imputação subjetiva deveria ou não trazer alguma consequência na hora de determinar si ao imputável é possível impor uma medida de segurança.

Palauras-chave: inimputabilidade; atribuição de dolo; erro de tipo psiquicamente condicionado; medidas de segurança. 


\section{INTRODUCCIÓN}

El objeto del presente artículo es analizar si determinadas circunstancias personales del autor, que usualmente son entendidas como casos de inimputabilidad, debieran ser consideradas por el juez a la hora de precisar si el agente actúa o no con dolo y los efectos que tal decisión podrían tener en sede de imposición de medidas de seguridad. Como se podrá analizar a lo largo de este trabajo, la solución al problema planteado no resulta sencilla y, al menos en Chile, la práctica judicial no ha transparentado, las más de las veces, los puntos de partida de la teoría del delito que asume para resolver tales supuestos. Con el propósito de clarificar esta situación se puede tener en cuenta un caso concreto para verificar así, las diversas opciones por las que podría optarse:

El día 29 de abril del año 2015, a las 23:00 horas aproximadamente, el imputado de nombre J. L. A. M. C. se constituyó en el domicilio de la afectada J. P. B. A., antejardín del domicilio ubicado en I.C.B N²951, San Antonio. Agregando que llegó gritando insultos a la afectada, incumpliendo la medida cautelar dictada en resolución de causa RIT 23942015 de fecha 24 de abril del año 2015 por el Tribunal de Garantía de esta ciudad, imponiendo como medida la establecida en el Art. 9 letra b) de la Ley 20.066, esto es, la prohibición de acercarse a la víctima a su domicilio, lugar de trabajo o cualquier otro lugar que concurra, la cual se encontraba vigente a la época de ocurrencia de los hechos y notificada en forma personal al imputado'.

De acuerdo a lo esgrimido por el Ministerio Público, los hechos antes descritos constituirían el delito de desacato, previsto y sancionado en el artículo 240 del Código de Procedimiento Civil chileno, se encuentra dicho ilícito en grado de consumado y le corresponde al acusado la calidad de autor.

Lo relevante del caso es que se constata en juicio que el acusado padecía de una enfermedad crónica con espectro esquizofrénico con deterioro psicoorgánico moderado que no le permitiría tomar decisiones y que, por tanto, no sería capaz de autodeterminarse ni de anticiparse a las consecuencias de su conducta.

A juicio del tribunal, su trastorno afectaría la mayor parte de las funciones cognitivas y síquicas, entre las más puntuales, memoria, etc., impidiéndole tomar decisiones. Por lo mismo, se concluye por parte del tribunal, que el acusado no habría actuado con dolo, debido a la falta de conocimiento y comprensión de la prohibición de acercamiento que se le impuso. Pese a lo anterior se resuelve imponer una medida de seguridad al sujeto debido que el tribunal habría podido constatar la peligrosidad del mismo. Frente a tal

\footnotetext{
Los hechos corresponden a la causa RIT 203-2016 y los razonamientos que se expondrán a continuación reflejan tanto la resolución del TOP de San Antonio, así como a la SCA Rol 2223-2016 (de 6 de enero de 2017), correspondiente a recurso de nulidad interpuesto por la Defensoría Penal Pública contra dicha sentencia por errónea aplicación del derecho.
} 
resolución, la Corte de apelaciones respectiva estimó que la resolución recurrida concluía sin mayores fundamentos, "que el hecho típico se encontraba plenamente establecido, aun cuando, al mismo tiempo, dieron por demostrado que el dolo (que llaman específico) no se encontraba presente por tratarse de un inimputable" (SCA, Rol 2223-2016, considerando sexto). A juicio de la Corte de apelaciones se advierte una errónea aplicación del derecho puesto que, dando por establecida la falta de conocimiento y comprensión con respecto a la prohibición vulnerada, desplazarían tales falencias hacia la culpabilidad, siendo que, en estricto rigor, debió haberlas considerado ya a la hora de determinar si se cumplían los requisitos del tipo penal —específicamente el tipo subjetivo-.

Como se puede apreciar, nos enfrentamos al dilema de si ciertas circunstancias personales del sujeto, habitualmente examinadas a propósito de la culpabilidad, y específicamente a propósito de lo que se entiende por imputabilidad —o capacidad de culpabilidad - debieran o no tener incidencia a la hora de configurar elementos previos del delito, específicamente en el caso concreto, el de la tipicidad subjetiva.

Para resolver el problema planteado, debemos tener en cuenta, en primer lugar, qué es lo que se entiende habitualmente por imputabilidad. Como ha señalado Cillero (2011): "para la doctrina nacional mayoritaria, la imputabilidad es la capacidad de conocer lo injusto y de determinarse según ese conocimiento $\mathrm{o}$, dicho sintéticamente, imputabilidad es capacidad de culpabilidad" (2011, p. 179). O como sostiene Garrido:

\begin{abstract}
Analizada desde el ámbito semántico, la expresión imputabilidad significa "atribuibilidad" o sea las diversas condiciones que debe cumplir un sujeto para ser susceptible de reproche, pero si bien esa noción corresponde a la idea, presenta un aspecto formal de lo que es imputabilidad, cuando lo que interesa es su noción substancial. Con distintas expresiones, la mayor parte de los autores concuerdan en que este último sentido se traduce en el siguiente concepto: "capacidad de conocer qué es injusto y de actuar conforme a tal comprensión" (2003, p. 208).
\end{abstract}

Sin lugar a dudas, para el objeto de estudio, los casos que parecen más problemáticos son aquellos en los que la condición personal del sujeto está dada por aquellos problemas de salud asociados a la pérdida de las condiciones físicas y mentales para percibir la realidad, comprenderla y actuar conforme a ella. Esto es, según Cavero (2012), de "enfermedades o situaciones de pérdida de las capacidades personales mínimas para poder recibir una imputación penal" (p. 643)².

En efecto, si el sujeto de la imputación, debido a su condición psíquica, no estaba en condiciones de conocer que su comportamiento realizaba los elementos de un tipo penal determinado, podrían no

\footnotetext{
2 Fundamental, y en el ámbito angloamericano se pueden considerar a Morse y Hoffman (2007, p. 1087), para quienes ciertos desórdenes mentales pueden impedir la capacidad de formación del requisito del mens rea.
} 
satisfacerse los requisitos del tipo subjetivo — doloso—, en la medida en que se entienda por dolo el conocer y querer la realización de los elementos de un tipo penal y que el agente haya actuado en lo que se conoce como un error de tipo psíquicamente condicionado-. Así las cosas, no se cumplirían los requisitos del tipo penal y, por tanto, en un sistema del delito compuesto por las categorías de la tipicidad, antijuridicidad y culpabilidad, la ausencia de estos impediría predicar el injusto de la conducta, por lo que tampoco tendría sentido la pregunta por la culpabilidad del autor.

\section{INCAPACIDAD DE CONOCER EL INJUSTO DEL ACTUAR: ¿PROBLEMA PROPIO DE LA CULPABILIDAD O DEBE INCIDIR EN LA CONFORMACIÓN DEL INJUSTO?}

Ya hace un buen tiempo Joshi (1989) sostuvo, en España, que para ciertos casos en los que los tribunales declararon inimputables a personas que cometieron un hecho típico, se debió haber considerado tales circunstancias personales - que sirvieron de base de la declaración de inimputabilidad - en forma previa, específicamente, en la configuración de injusto. Como indica Silva, "las alteraciones psíquicas, en determinados casos, pueden no limitarse a afectar la culpabilidad del sujeto, excluyendo o atenuando su imputabilidad, sino que, además, pueden afectar a la acción, la tipicidad o la antijuridicidad de muy diversas formas, directas o indirectas" (2003, p.100). El autor distingue cuatro supuestos de casos: a) Aquel en el que la alteración psíquica provoca un error acerca de la concurrencia de elementos fundamentadores de la responsabilidad efectivamente existentes (ausencia de acción, error de tipo).

b) Aquel en el que la alteración psíquica produce la suposición errónea de la concurrencia de los elementos de una causa de justificación o exclusión de la culpabilidad (eximentes putativas).

c) Aquel en el que la alteración psíquica provoca la suposición errónea de la concurrencia de elementos fundamentadores de la responsabilidad (tentativa irreal o tentativa inidónea, según la concepción de la tentativa que se acoja).

d) Aquel en el que alteración psíquica da lugar a un error sobre la concurrencia de situaciones justificantes o exculpantes realmente existentes (casos de falta del elemento subjetivo de justificación o de exculpación). (Silva, 2003, p. 101).

Habría sido habitual, según el autor, que en tales supuestos se sostuviera por cierto sector de la doctrina la necesidad de aplicar medidas de seguridad en caso de verificar la peligrosidad del sujeto, pero que se adujeran, al mismo tiempo, dificultades dogmáticas, e incluso legales, para tal aplicación (Silva, 2003, p.101).

Como se podrá apreciar a continuación, uno de los principales problemas de índole sistemático que se habrían planteado 
es el de determinar si la concurrencia de tales circunstancias puede incidir o no en la respuesta a si es posible hablar de comportamiento doloso. Lo anterior se debe a una razón bastante sencilla: si bien es cierto que la imputabilidad implica capacidad de conocer el injusto del actuar, en aquellos casos de alteración de la percepción de la realidad o de mera incapacidad de comprensión, también lo es que podría plantearse la legítima duda de si esa "incapacidad de conocer" podría afectar inicialmente la posibilidad de que se satisfagan ciertas exigencias provenientes del tipo subjetivo. Por lo mismo, debemos ahondar brevemente en algunos conceptos previos, esto es, los del dolo y su exclusión.

\subsection{El dolo: contenido del dolo y su exclusión}

Como ha señalado Hernández (2011), nuestra doctrina ha entendido el dolo desde una perspectiva dual, esto es, como conocimiento y voluntad de realización del hecho típico. Esto quiere decir, al menos, y siguiendo en esto a Kindhäuser, que un sujeto actúa dolosamente "si en atención a las circunstancias objetivas del hecho relevantes, obra con el grado de conocimiento, y dado el caso con la disposición volitiva, que requiere la definición objetiva de dolo que ha de ser aplicada" (2008, p. 5). Tal punto de partida nos permite, además, clarificar la relación que se presenta entre lo que se conoce como tipo objetivo y tipo subjetivo, ya que, de acuerdo con este autor, tal relación no responde a elementos de un mismo orden lógico, sino de otro orden: "los elementos del tipo subjetivo se encuentran referidos, precisamente, a los elementos del tipo objetivo" (Kindhäuser, 2008, p. 5) o, en otras palabras, "al tipo objetivo pertenece el acontecer que se le reprocha al autor" (Kindhäuser, 2006, p. 65), lo que corresponde, a su vez, al contenido de la imputación subjetiva compuesto, en definitiva, por los conocimientos del autor como fundamento y el límite de la imputación —dolo e imprudencia-.

Como es bien sabido, la doctrina penal suele diferenciar diversas formas de dolo, atendiendo a si, junto a los elementos cognitivos, priman elementos volitivos, como en el dolo directo de primer grado, el que es conocido como "intención", en contraposición a lo que se conoce como dolo eventual, construido principalmente sobre la base de puros elementos cognitivos (Cury, 2005; Garrido, 2003; Politoff, Matus y Ramírez, 2003).

Con todo, es importante considerar que se ha puesto en duda la posibilidad de equiparar el elemento volitivo al cognitivo. Así, por ejemplo, para Hruschka (2005) entre ambos existiría una relación de dependencia de acuerdo con la cual "siempre que un autor lleva a cabo una determinada conducta, conociendo el carácter de la acción y las circunstancias, quiere también realizar tanto la conducta como las circunstancias en cuestión" (p. 148). Con independencia de lo anterior, lo que no puede ser puesto en duda es que lo que es común a toda forma de dolo, es decir, aquello que constituye su contenido mínimo, es su componente cognitivo. Como indica recientemente Sánchez-Málaga (2015): "el dolo debe 
implicar, por lo menos, la realización de un hecho constitutivo de infracción penal, con correcto conocimiento de las circunstancias que integran el tipo de dicha infracción" (p. 63).

El análisis de este contenido mínimo del dolo resulta fundamental para el objeto del presente artículo, en la medida en que lo que interesa es determinar las condiciones bajo las cuales un sujeto habría actuado desconociendo que su comportamiento realiza los elementos de un tipo de delito. Por tal razón, debemos ahondar brevemente en el alcance del conocimiento propio del dolo.

Según una aproximación que ya resulta minoritaria en Chile, el dolo podría ser entendido como conocimiento de la realización de un delito, lo que supondría también la consciencia de la antijuridicidad o del carácter prohibido de ese hecho. Así, por ejemplo, Etcheberry (1998), para quien "dolo es el conocimiento de los hechos constitutivos del tipo, acompañado de la conciencia de su antijuridicidad y la intención o aceptación de su posible resultado" (p. 292), o Novoa, quien sostuvo que el dolo es un elemento de la teoría del delito que debe examinarse en la esfera de la culpabilidad, lo que se debía a que "el dolo, al igual que la culpa, es una de las especies de culpabilidad que permiten fundamentar el reproche jurídico subjetivo" (2005, p. 465).

Como indica Luzón, el dolo se concebía como dolo subjetivamente malo (dolus malus) y, por ello, resultaba coherente su ubicación dentro de la culpabilidad "como grado superior o forma más grave, pues para la plena culpabilidad (individual) es preciso el conocimiento de la prohibición o antijuridicidad por parte del sujeto" (2016, p. 386). En este sentido, es importante considerar que el dolo era entendido, por la teoría clásica del delito, como una forma de culpabilidad, que se comprendía como una reunión de todos los requisitos subjetivos del delito y se caracterizaba, de acuerdo a Luzón por constituir un concepto meramente descriptivo:
Concretamente como comproba- ción de un nexo psicológico, no material, entre el autor y el hecho. Lo característico del dolo sería que el nexo psíquico que une al autor con el hecho es la voluntad o incluso intención, ya que el sujeto conoce y quiere realizar el hecho (causar el resultado en los delitos de resultado). (Luzón, 2016, p. 219).

No obstante, en la actualidad se considera como mayoritaria, al menos en la doctrina, aquella concepción que ubica al dolo en el injusto, lo que se explica por diversas razones. Así, por motivos que conciernen a la propia categoría de la culpabilidad y que se explican por el proceso de normativización de la misma, se ha ido abandonando la división objetivo-subjetiva del delito característica del concepto causalista del delito y que justificaba la consideración del dolo y la imprudencia como expresión de "puras relaciones psicológicas hacia un injusto definido en términos objetivos" (Sánchez-Málaga, 2015, p.63). Por otra parte, con la primacía del concepto personal de 
injusto no parece correcta la consideración de este elemento del delito sin consideración a las posibilidades de evitación del menoscabo de bienes jurídicos. De acuerdo a Kindhäuser

\begin{abstract}
Ya el juicio de antijuridicidad, el cual antecede a la imputación de culpabilidad, no podrá más que referirse a aquellas formas de comportamiento que pueden y deben ser evitadas (tratándose de prohibiciones) o ejecutadas (tratándose de mandatos) para evitar el menoscabo de un bien jurídico. (Kindhäuser, 2008, p.7).
\end{abstract}

Por lo tanto, se entiende que no pueda ser, a su vez, considerado como jurídico penalmente relevante aquellas afectaciones a bienes jurídicos, que "no son evitables a través del seguimiento de una norma" (Kindhäuser, 2008, p. 7).

Una de las principales consecuencias de tal diferenciación es el abandono de la concepción del dolo malo, y la reducción del conocimiento del dolo entendido como dolo natural. Por tanto, vinculado exclusivamente a los elementos objetivos del tipo penal, sin necesidad de ahondar en el problema del conocimiento de la antijuridicidad, el que se transforma en un asunto que atañe a la categoría de la culpabilidad.

Así las cosas, parece más fácil abordar el contenido cognitivo del dolo. Como indica Hernández, existiría acuerdo en cuanto a que el dolo "requiere que el sujeto advierta en su conducta y en las circunstancias en que ésta se desarrolla todas las notas que son relevantes desde el punto de vista del tipo penal" (2011, p. 54). Con todo, tal aseveración puede ser evidentemente problematizada en la medida en que, dependiendo del alcance que se le asigne a la categoría de la tipicidad, también variará el contenido del tipo subjetivo. De acuerdo a Luzón (2016), no existiría acuerdo respecto al contenido de la categoría "elementos objetivos del tipo", ya que, por una parte, se puede entender exclusivamente referida al tipo indiciario -o conocido como tipo positivo o estricto- - o, por la otra, junto a lo anterior al conocimiento de la ausencia de los elementos objetivos de las causas de justificación. Este problema resulta relevante, sobre todo, para efectos de delimitar la exclusión del dolo por casos de error.

Una vez que se han descrito algunos aspectos centrales relativos a lo que se ha entendido por dolo, se debe abordar el problema de las consecuencias asociadas a la constatación de que el sujeto ha obrado desconociendo que su comportamiento realizaba los elementos objetivos de un tipo penal. Este paso sería del todo relevante para la configuración del injusto en un sistema de injusto personal, ya que, la ausencia de tal conocimiento implica la ausencia del dolo y, por ende, que no se satisfagan las exigencias asociadas a la tipicidad subjetiva, salvo en ciertos casos en que subsistiría la opción de imputar subjetivamente a título de imprudencia. Como señala acertadamente Fakhouri (2009): "dolo y error constituyen las dos caras de una misma moneda. Por este motivo, la decisión acerca del error que excluye el dolo y del error que lo deja 
intacto se encuentra estrechamente vinculada con el concepto de dolo que se maneje" (p. 5).

Así, nuestra doctrina y jurisprudencia reconocen que la ausencia de conocimiento, es decir, el error sobre cualquier elemento del tipo, excluye el dolo, sea evitable o no tal desconocimiento por parte del autor (Cury, 2005, p. 309).

La clase de desconocimiento que interesa para la configuración del tipo subjetivo es aquella que, en definitiva, versa sobre el objeto de la valoración, esto es, la conducta del sujeto en sus circunstancias concretas, y no el desconocimiento del carácter antijurídico de tal conducta, conocido como error de prohibición, que consiste en un error acerca de la valoración del objeto - la misma conducta - y que en la actualidad se suele abordar como un asunto propio de la culpabilidad.

Así, si el agente desconoce que está manteniendo relaciones sexuales consentidas con menor de 14 años, por creer, en forma evitable o inevitable, que el otro tiene 18 años, no actúa con dolo en la medida en que yerra acerca del objeto que se valora como antijurídico, cree estar realizando una conducta diversa de aquella des valorada. Desconoce que su conducta realiza los elementos del tipo penal del artículo 362 del Código Penal chileno. Por el contrario, si sabe que la menor aún no cumple 14, pero cree que tal conducta no se encuentra prohibida, desconocería la valoración del objeto, esto es, su carácter de antijurídico.
Este punto resulta fundamental en la medida en que, como se podrá apreciar a continuación, una de las alternativas que se ha planteado por alguna doctrina sería que en ciertos supuestos de sujetos que padecen determinados trastornos mentales - usualmente analizados a propósito de la culpabilidad — se tendría que asumir que el sujeto no estaba en condiciones en razón del trastorno en particular-de conocer que su comportamiento realizaba los elementos de un tipo.

\section{2 ¿Ausencia de dolo en casos de error psíquicamente condicionado?}

Excluir el dolo en tales casos no es irrelevante desde el punto de vista de las consecuencias prácticas, ya que dependiendo del lugar que se le asigne y de su rol en la configuración del injusto, incluso se podría llegar a la conclusión de que se está en presencia de un comportamiento que no contraviene al ordenamiento jurídico. Molina (2008), entre otros, ha señalado que lo anterior es de suma importancia, en la medida en que tal conclusión traería efectos asociados a las posibilidades de defensa — por parte del tercero afectado por el ataque del agente que actúa en tal situación de desconocimiento-, o incluso en la posibilidad de dictar medidas de seguridad si se entiende que las mismas solo pueden ser aplicadas en caso de que se constate en forma previa un hecho típico y antijurídico y se interprete tal exigencia en forma equivalente al "injusto personal" y, al mismo tiempo, como podremos apreciar, se considere que la configuración del tal injusto supone acreditar — en el sentido de demostrar — que 
el sujeto actuó, efectivamente, con dolo como un problema psíquicamente o sicológicamente constatable. Por ejemplo, a través de indicios que permitan extraer de su comportamiento externo que conocía la realización de determinada conducta y no otra.

Piénsese en el siguiente caso: un sujeto, que padece una esquizofrenia con alucinaciones acústicas, ingresa a un centro comercial y toma un bien mueble ajeno creyendo - producto de esta clase de alucinaciones - que era suyo debido a que se la habían regalado los dueños de la tienda, quienes le habían informado de tal situación por los parlantes del establecimiento. Como se puede apreciar, tal caso se podría distinguir de otro grupo de casos diversos en los que el trastorno mental puede llevar a una persona con alucinaciones a matar a un sujeto viendo en él el rostro de su enemigo. O frente a los casos en que un sujeto mata a los hijos de su enemigo debido a que, en un delirio, cree que es la única forma de evitar la llegada del anticristo a vivir entre los hombres. Es evidente que, en estos dos supuestos, quien mata sabe que está matando a otro (Morse y Hoffman, 2007, p. 1089-1090).

En este sentido, Joshi (1989), cuestiona la práctica de los tribunales que aplican una medida de seguridad sin verificar, previamente, si concurren los elementos del injusto, ya que se remitirían exclusivamente a centrarse en la eventual exclusión de responsabilidad por inimputabilidad. En el fondo cuestiona que, para este grupo de supuestos, no se ponga en duda la presencia de un comportamiento doloso debido a la concurrencia de un error de tipo invencible. En Latinoamérica, cierta doctrina habla derechamente de "error de tipo por incapacidad psíquica", así, por ejemplo:

El agente puede incurrir en errores de tipo por incapacidad psíquica permanente o transitoria: quien por alteración morbosa de las facultades o por insuficiencia de éstas (...) no tiene, en el momento de la acción, la capacidad de conocer o de actualizar los elementos conscientes necesarios para configurar la finalidad realizadora del tipo, no obra con dolo. No se trata aquí sólo de supuestos de autismo esquizofrénico y alteraciones de la sensopercepción (ilusiones y alucinaciones) que impidan reconocer los elementos objetivos del tipo (quien percibe animales y son personas) sino también: (a) los oligofrénicos que no pueden comprender ciertos conceptos abstractos, como la ajenidad de la cosa; (b) los niños pequeños, respecto de los cuales es difícil pretender que conceptúen como cosa algunos objetos; (c) las personas que obran bajo los efectos del miedo grave (pánico) que les perturba la sensopercepción o la actualización de algunos conocimientos; (d) las personas que por efecto del cansancio y la falta de sueño sufren alteraciones de la sensopercepción. (Zaffaroni, Alagia y Slokar, 2002, p. 535).

Tal cuestionamiento, de atribuir comportamiento doloso, se basaría en el hecho de que, en el caso planteado, se trataría de un supuesto en el que el agente: 
Como consecuencia de su enfermedad o trastorno psíquico (...) ve menguada su capacidad de percepción de la realidad, o desconoce leyes causales del actuar humano, o criterios de significación social, de modo que, en definitiva, ignora el contenido último de lo que hace. (Silva, 2003, p. 103-104).

Ahora, debido a que el objeto del artículo dice relación con detectar exclusivamente la incidencia de la constatación de tales alteraciones en la configuración del tipo subjetivo, específicamente en lo que atañe a la atribución de dolo, nos remitiremos exclusivamente a los supuestos que sean relevantes desde esta perspectiva y a sus consecuencias para la imposición de medidas de seguridad.

Según lo señalado por parte de la doctrina, el problema que se podría presentar acá es que, frente al mismo caso, un sujeto que no padezca tal alteración quedaría impune (en caso de errar sobre tal circunstancia) mientras que, al inimputable, habiendo "incurrido en el mismo error", se le aplicaría una medida de seguridad (Joshi, 1989, p.127) . Por tal razón, este mismo autor, propone distinguir una serie de situaciones para evitar así arribar a resultados que califica como poco satisfactorios. En primer lugar, señala que se podría dar el caso de que un inimputable o un sujeto con imputabilidad disminuida termine llevando a cabo una conducta de la cual no se pueda predicar su antijuridicidad — sea por ausencia de acción, por concurrir un error de tipo in- vencible o por la "presencia de una causa de justificación" (Joshi, 1989, p. 128)—, pero en donde, en los mismos casos, un "espectador objetivo" se hubiera comportado del mismo modo. Así, por ejemplo, si A, que es un "enfermo mental", confunde a B con un jabalí, dispara y lo mata, pero un "espectador objetivo" hubiera cometido el mismo error —utilizando un pronóstico ex ante-. En tales supuestos, como propone Joshi (1989), no se podría aplicar una medida de seguridad, ya que se transgrediría el principio de legalidad y, además, porque se terminarían confundiendo plenamente las medidas predelictuales con las posdelictuales.

Un segundo grupo de casos estaría dado por situaciones en las que la inimputabilidad del autor sea, precisamente, la que condicione el desconocimiento de la situación y, por tanto, a diferencia del primer grupo de supuestos, un espectador objetivo, puesto en la posición del agente — solo en un sentido externo y sin considerar su individualidad, esto es, como señala la autora "los conocimientos objetivables del autor"-, hubiera estado en condiciones de conocer que realizaba los elementos objetivos de un tipo penal. Para graficar esta situación propone el siguiente ejemplo: "A, que debido a una enfermedad mental cree que todos los abrigos de piel que se encuentra son regalos de Dios, y que, por tanto, está legitimado a tomarlos" (Joshi, 1989, p. 131). Sostiene que, en este caso, no puede ponerse en duda la "presencia de un error de tipo, que pudiera ser incluso invencible — error sobre la ajenidad de las cosas - " (Joshi, 1989, p. 131). 
De acuerdo a Joshi (1989), en tal grupo de casos, y a diferencia del anterior, un espectador objetivo habría estado en condiciones de conocer la ajenidad de la cosa, por lo que el error se trata de uno psíquicamente condicionado. Como bien señala Silva (2003), esta aproximación asume que puede distinguirse entre errores condicionados por la anomalía frente a errores que no lo estarían. Señala que, por regla general, "parece que se asume el criterio de que no estaría condicionado por la enfermedad aquel error en el que habría incurrido también un sujeto sano (se entiende que un sujeto sano "medio"). Con todo, señala que esta aproximación podría no ser correcta, ya que "tal vez no debiera partirse de un indeterminado sujeto "medio", sino de si el mismo sujeto, de no sufrir la alteración o anomalía en cuestión, habría incurrido o no, dados los demás factores de su personalidad, en tal error" (Silva, 2003, p. 112).

Junto con lo anterior, Joshi (1989) señala que en este caso sería evidente que se trataría de sujetos peligrosos, por lo que, desde una perspectiva de política criminal, parece adecuado aplicarles una medida terapéutica: "En efecto, un sujeto que, debido a su enfermedad mental, incurre sistemática y repetidamente en un error de tipo cuando se dan ciertas circunstancias (exteriores o interiores) puede ser un sujeto «peligroso»" (Joshi, 1989, p. 131).

Resulta evidente que este problema solo se presenta si se asume que la ausencia de dolo trae consecuencias para la configuración del injusto (Silva, 2003, p. 126; Silva, 2014, p.3). Por ende, si se considera que la ausencia de dolo es una cuestión que, a lo más, excluye la culpabilidad del sujeto debido a que no se ha constatado la respectiva relación psicológica interna entre el autor y el hecho en particular, lo anterior no impediría aplicar, en su caso, una medida de seguridad.

Por el contrario, y como señala Joshi (1989), quien incluya el dolo y la imprudencia en el injusto "deberá exigir su presencia como presupuesto para la aplicación de una medida" (p. 133), por lo que, en estos casos, no "será posible aplicar medida de seguridad alguna al imputable que actúe en error de tipo invencible" (Joshi, 1989, p. 133).

Es importante considerar que la autora arriba a tal conclusión debido a que el artículo 6 a) del Código Penal vigente a la época, consagraba que para determinar la vencibilidad del error de tipo hay que tener en cuenta las «circunstancias del hecho y las personales del autor». En la actualidad el artículo 14 número 1, del Código Penal español señala que: "si el error, atendidas las circunstancias del hecho y las personales del autor, fuere vencible, la infracción será castigada, en su caso, como imprudente". Como se puede ver, la autora parte de la siguiente premisa: para el tipo subjetivo, la normalidad o anormalidad motivacional es, precisamente, una circunstancia personal relevante no solo en sede de culpabilidad. Por otra parte, también se debe tomar en cuenta que las circunstancias han cambiado en relación a las exigencias para la imposición de medidas de seguridad desde el tiempo de esta publicación. En su tiempo, 
el problema interpretativo consistía en determinar qué se entendía por "hecho que la ley sancionare como delito", en cambio, en la actualidad el artículo 6.1 del Código Penal español señala que "las medidas de seguridad se fundamentan en la peligrosidad criminal del sujeto al que se impongan, exteriorizada en la comisión de un hecho previsto como delito". Tal diferenciación podría ser relevante, como se podrá ver más adelante, para explicar cierta tendencia de la doctrina española a reconocer que, pese a que el sujeto no actúa con dolo para estos casos y que, por tanto, no se satisfarían las exigencias del injusto personal, igualmente se podrían aplicar medidas de seguridad para el caso de verificarse la peligrosidad.

Como se puede apreciar, esta postura parte del supuesto de que la invencibilidad es un criterio que se debe determinar de acuerdo a parámetros personales del autor y no bajo criterios normativos - es decir, bajo un estándar objetivo-, ya que, "el conocimiento de los hechos se obtiene a través de una experiencia subjetiva, que por tanto difiere de cada sujeto" (Joshi, 1989, p. 134). Presumir el dolo en el sujeto que actúa en error psíquicamente condicionado transgrediría, así, el principio de culpabilidad (Joshi, 1989, p.135). Al respecto, Molina describe una serie de posturas que, en forma similar, adhieren a la ilegitimidad de la imposición de tales medidas, ya que "si falta el dolo típico u otros elementos subjetivos del injusto no hay ninguna razón para intervenir con medidas, con independencia de cuál sea la razón que ha provocado dicha ausencia" (Molina, 2008, p.124).
Si bien es cierto, esta posición podría reforzar la corrección del razonamiento de la Corte de apelaciones puesto como ejemplo en un comienzo, es necesario considerar algunas de sus principales debilidades. Lo primero que se debe tener en cuenta es que este argumento supone, en primer lugar, que una actuación mediada por una circunstancia personal que impide conocer que se realiza los elementos de un tipo penal — como es la enajenación mental—, es ya relevante en sede de tipicidad subjetiva. Lo segundo, que supone que la consecuencia que se extrae de lo anterior es que no se cumplirían los requisitos para aplicar una medida de seguridad ya que, para la misma, se exige la presencia de un hecho típico y antijurídico, entendiendo acá tal requisito en forma equivalente a "injusto personal".

Por lo mismo, una respuesta posible frente a la alternativa de negar la procedencia de medidas de seguridad luego de la verificación de tales circunstancias personales que impiden conocer la significación de la conducta, es la de negar su incidencia en sede de injusto, debido a que estas serían un problema propio de la culpabilidad. Silva (2003) describe claramente tal tendencia de la siguiente forma: quienes no están dispuestos a prescindir de elementos subjetivos para la afirmación de la antijuridicidad necesaria para la aplicación de medidas de seguridad, terminan defendiendo la legitimidad de su imposición a través de argumentos bastante evasivos. Así, por ejemplo, el sostener que en sede del injusto los errores psíquicamente condicionados no deben ser considerados, pero sin dar 
cuenta realmente de los motivos por los cuales tal exclusión resultaría correcta. En efecto, para negar la atipicidad de tal clase de comportamientos, como ya se ha descrito, se ha recurrido, sin más, a la distinción entre errores condicionados por la anomalía o alteración psíquica y los errores no condicionados por las mismas, ya que solo en el caso de los segundos, "aquellos en los que también una persona normal hubiera errado, el efecto eximente se produce por la vía del error, sin esperar a la inimputabilidad, mientras que en los primeros el error sería irrelevante" (Molina, 2008, p. 128).

El problema es que no se trasparentan los diversos e incompatibles puntos de partida que están detrás de tal diferenciación entre el sujeto normal —o espectador objetivo- - y el sujeto que padece de un trastorno mental que condiciona su error. Por un lado, parecen recurrir a una perspectiva psicológica para la determinación del dolo, donde lo que se requiere para tales efectos es de la acreditación de un hecho psicológico del auto. Para esta perspectiva, de acuerdo a Ragués (2002), en el proceso de determinación del dolo se debería averiguar una realidad que se encuentra en la cabeza del autor, basándose para esto en vivencias subjetivas del autor al momento del hecho, fenómenos a los que se puede y debe acceder en el momento posterior del proceso. Pero, por otra parte, quienes proponen la diferenciación ya descrita, acudirían, a su vez, a un criterio atributivo de índole normativo - que es lo que conocería, ex ante, un sujeto normal-. En definitiva, no se configuraría el injusto debido a que no se considera la individualidad psíquica del sujeto, más bien se excluye debido a que se recurre a un parámetro normativo, como es el de las posibilidades de conocimiento del espectador objetivo puesto en el lugar del agente y, obviamente, sin considerar su trastorno mental.

Por lo tanto, para excluir el comportamiento doloso en estos casos, bastaría, simplemente, con constatar que este espectador objetivo, puesto en la situación del agente - esto es, sus circunstancias personales externas - no estaba en condiciones de conocer y, por ende, no podría atribuírsele al mismo haber actuado con dolo. Como indica Silva (2003), recurrirían a criterios normativos para la satisfacción del tipo subjetivo, a pesar de "su evidente ausencia empírica" (p.127). Pero lo que no llegan a explicar realmente es por qué razón para la conformación del tipo subjetivo y, por ello, del injusto, se podría prescindir de consideraciones personales — de la individualidad- del sujeto y bastaría la referencia a criterios normativos como el del "sujeto normal". Como acertadamente señala cierta doctrina, si no se dan mayores razones para defender esta solución parecería que el dolo "o los elementos subjetivos del injusto ya no serían siempre elementos necesarios de la tipicidad, sino contingentes. Podría prescindirse de ellos precisamente en aquellos casos en los que no están presentes debido a la inimputabilidad del autor" (Molina, 2008, p.129). Por lo mismo, otra alternativa criticada por esta postura sería la de cambiar la definición de dolo "por ejemplo, aceptando que sería doloso también el comportamiento de quien 
desconoce los elementos objetivos del tipo, pero en una situación en la que un hombre normal, en el que no concurran circunstancias de inimputabilidad, no hubiera incurrido en dicho desconocimiento" (Molina, 2008, p. 130), lo que, para tal autor significaría destruir el núcleo básico de la imputación subjetiva.

Por esta misma razón, la respuesta al problema planteado supone dar cuenta, en forma previa, de qué se quiere decir cuando se sostiene que el autor ha obrado con dolo. Es decir, si lo que está detrás para tal aseveración es un proceso de acreditación de un hecho interno - psicológico - o más bien, un proceso atributivo o de imputación de conocimiento. El punto es del todo relevante, ya que, si se parte de la base de que para la afirmación del dolo se debe acreditar o constatar tal relación subjetiva efectivamente dada entre el sujeto y el suceso, resulta evidente la consecuencia para el problema planteado. Frente a la acreditación de una enajenación mental del sujeto a partir de la cual sea evidente que no estuvo en condiciones de conocer el significado de su comportamiento - por ejemplo, que la cosa que tomaba era ajena, o que a quien mataba era "otro"-, no tendría mucho sentido prescindir de tal información para luego considerarla recién en sede de culpabilidad.

En la esfera procesal, lo anteriormente dicho tendría como correlato, principalmente, el problema de la "acreditación del dolo", es decir, el de la prueba del mismo. Como de lo que se trata es de averiguar qué pasaba en la mente del autor al momento de la comisión del hecho en particular, el desafío que se ha tratado de resolver es el de los "datos externos" o del contexto que permitirían concluir que el agente ha actuado con dolo. Al respecto, Ragués (2002), ha señalado que esta tendencia ha recurrido, principalmente, a la prueba indiciaria para la determinación de la concurrencia de los procesos psíquicos sobre los que se asentaría el dolo. El autor señala, eso sí, que tal proceder requiere del cumplimiento de ciertas condiciones:

Sólo puede garantizarse que la conclusión obtenida mediante la práctica de la prueba de indicio vaya a coincidir con la realidad efectivamente acaecida si se respetan dos condiciones irrenunciables: en primer lugar, debe contarse con una regla de experiencia de vigencia incontrovertida que explicite bajo qué condiciones es segura la existencia de un determinado grado de conocimiento en una persona; en segundo lugar, los hechos previamente probados que, a modo de premisa menor, se vinculen a dicha regla de experiencia, no pueden suscitar ningún género de dudas en cuanto a su plena coincidencia con la realidad. (Ragués, 2002, p. 257).

Así, por ejemplo, en forma bastante expresa se ha pronunciado la Corte Suprema de Chile: Que si bien la prueba del dolo es una
de las cuestiones más problemáticas
en la sede procesal penal, la deter-
minación acerca de su concurrencia
ha de basarse en precisas circuns-
tancias —anteriores, coetáneas
y posteriores al hecho, de todo
orden-que demuestren al exterior 
el íntimo conocimiento y voluntad del agente, toda vez que, como todo lo que se guarda en lo más profundo de la psiquis del individuo, sólo puede probarse por una serie de datos que manifiesten la intención requerida. Por consiguiente, el dolo, elemento subjetivo necesario para imponer una sanción penal, solamente puede conocerse y fijarse por un proceso de inducción. Estos juicios de inferencia — como actualmente se prefiere denominarlospermiten al tribunal, mediante una operación lógica, deducir del material fáctico que la probanza practicada ha puesto a su alcance la concurrencia de los componentes anímicos del suceso delictuoso, ya que el objeto de la convicción del tribunal es un elemento que en un principio permanece reservado en vivencias subjetivas del autor en el momento del hecho, de modo que para su averiguación, se requiere de una inferencia a partir de los datos exteriores. La falta de prueba directa acerca de un concreto estado de la conciencia o de la voluntad, lleva a la denominada prueba de indicios, para, a través de ciertos datos exteriores completamente acreditados, inferir la realidad de este estado de espíritu del autor de la infracción penal, necesario para la incriminación del comportamiento de que se trate. En definitiva, salvo espontáneo reconocimiento, el dolo ha de inducirse, lícita y racionalmente, de cuantas circunstancias giran alrededor —antes, durante y después de la conducta enjuiciada(SCS, 22 de abril de 2013).

Así las cosas, no cabría más que concluir que, en tales casos, parece indiscutible que ciertos estados mentales debieran ser considerados por el tribunal a la hora de determinar si el sujeto actúa o no en forma dolosa, en la medida que corresponde a datos del contexto que, si bien pertenecen a la individualidad del autor, no se ve por qué razón —al menos si se sigue esta tendencia- debieran omitirse a la hora de determinar si el sujeto actúa o no con conocimiento del significado de su conducta y no, por el contrario, en error de tipo. Que acá el error se presente condicionado por una anomalía psíquica en nada influye para arribar a la conclusión que debiera interesar: la constatación de actuación bajo la ausencia de conocimiento típicamente relevante.

Con todo, esto no supone necesariamente asumir que de lo que se trata en el tipo subjetivo es de acreditar tal conocimiento por parte del autor como si fuera un proceso de verificación de una relación psicológica del autor con el hecho. Actualmente, tal punto de partida ha sido fuertemente criticado por parte un sector de la doctrina, ya que supondría que la proposición "por la que se califica una conducta como dolosa" sería más bien descriptiva (Paredes, Díaz y de Vicente, 2001, p. 339). Es decir, y de acuerdo con Hruschka (2005), supone que el dolo es un hecho. Por el contrario, señala que el dolo no puede ser tratado como un hecho, ni siquiera como un hecho interno, y que lo que "realmente subyace a la afirmación de que un sujeto ha actuado dolosamente", no es una constatación o acreditación, sino que más bien surge de un proceso de imputación (Hruschka, 2005, p. 155).

Esto quiere decir que se debiera negar que el proceso de conformación del tipo 
subjetivo suponga la constatación efectiva, al menos como lo propone la perspectiva psicológica ya descrita, de una relación psicológica entre el autor y el hecho. Como sostiene García:

Tampoco se trata de deducir de los datos externos la interioridad de la persona. Esta comprensión disfraza la realidad del procedimiento de determinación del conocimiento del que el autor dispone, pues no se verifica ningún conocimiento psicológico, sino que el conocimiento necesario para el dolo se imputa al autor con base en criterios de referencia sociales asumidos por el Derecho penal (2012, p. 493).

Por lo mismo, tal sector de la doctrina - que ya cuenta con exponentes en Chile- considera que para tales efectos se recurre más bien a un proceso de imputación, es decir, como señala Mañalich (2005), se trataría de un juicio adscriptivo.

De acuerdo a Ragués (2002), la denominación genérica de "concepción normativa del dolo" se encontraría integrada por los autores que niegan la posibilidad de entender que la afirmación del dolo dependa de la averiguación de una determinada realidad psicológica y agregan que cuando el juez afirma que "alguien ha conocido algo" lo que hace es atribuir ese conocimiento al sujeto en virtud de determinados criterios que no pueden, o ni tan siquiera pretenden, constatar cuál fue el estado real de la subjetividad en el momento de la realización del hecho típico. Como resulta evidente, esta aproxima- ción supone necesariamente dar cuenta de los parámetros normativos — es decir, los criterios - a los cuales se recurre para atribuir el dolo y que lo anterior no resulte en un juicio arbitrario (Hruschka, 2005, p.155). Como señala Ragués (2002), la dificultad de esta postura radica en la legitimidad, ya que debe legitimar la utilización de criterios de imputación subjetiva que, de modo paradójico, renuncian explícitamente a la auténtica subjetividad del autor y además deben aportar una caracterización plausible de esos criterios.

Al respecto, lo primero que llama la atención es que parece no existir acuerdo en lo que se refiere a si para efectos de llevar a cabo tal proceso de atribución se deben tomar o no en cuenta las circunstancias personales objeto de estudio y en qué medida. Así, por ejemplo, para Ragués (2004), en el ámbito de la atribución del dolo no se operaría ni con un modelo puramente objetivo — como el del hombre medio propio de la imputación objetivani con uno que tuviera en cuenta al acusado individualmente considerado, con:
Todas sus anomalías y limita- ciones personales - como en la culpabilidad-, sino que se recurriría a un tercer modelo resultante de contextualizar al hombre medio en el escenario del concreto acusado, añadiéndole buena parte de sus característi- cas personales, pero sin tener en cuenta todavía determinados fac- tores de su individualidad, como, por ejemplo, las enfermedades psíquicas que pueda padecer. (Ragués, 2004, p.21). 
De acuerdo al autor, entonces, en el ámbito de la prueba de los elementos subjetivos —o más bien, de su atribución-. no se pretendería una "plena subjetivización del acusado", sino que se tomarían en cuenta aquellas circunstancias personales externas que permitirían acudir a lo que uno - quien efectúa el juicio adscriptivo- "habría pensado, visto, sentido o creído en esta situación concreta" (Ragués, 2002, p. 22).

En cambio, y solo a modo de ejemplo, para Paredes, Díaz y de Vicente (2001), el dolo también debiera ser entendido como atribución de sentido y, para tales efectos, no sería necesario "verificar empíricamente la existencia de dichos conocimientos, sino que bastaría con comprobar que el sujeto se encontraba de hecho en una situación, tanto personal como circunstancial, en la que, conforme a las «leyes de la experiencia», había de tenerlos" (p. 340). Parecería entenderse, de esta forma, que en el juicio adscriptivo se debiera tener en cuenta la realidad individual del autor, si es que se constata que debido a esta no parece adecuado atribuirle una situación de anormalidad relativa a su capacidad cognoscitiva.

Por su parte, García (2012), que también asume una perspectiva normativa de la determinación del dolo, sostiene que los criterios de atribución del conocimiento se asientan en la idea del rol y la persona del autor. Esto es, "para determinar el conocimiento del autor debe partirse de las competencias de conocimiento que cada rol impone a su titular" (2012, p. 494). Con todo, reconoce que para afirmar el dolo no bastaría constatar la "existencia de tales competencias de conocimiento", ya que también es necesario "que el autor haya podido alcanzar esos conocimientos en sus circunstancias personales, pues de otra manera la imputación personal no estará personalizada" (p. 494).

Como se puede observar, las consecuencias de adherir a una u otra alternativa serían sumamente diversas debido a lo disímiles que resultan los criterios atributivos o parámetros desde los cuales se debiera imputar a un sujeto que ha actuado con conocimiento. La diferencia se torna evidente si, por ejemplo, se recurre a un parámetro generalizador - como el de un hombre medio empírico-y, por tanto, no se consideran las circunstancias personales del agente, o a criterios individualizadores pero que, en definitiva, indican que tal imputación supone tomar en cuenta las circunstancias personales del sujeto. A su vez, también se podrían distinguir, dentro de esta última tendencia, diversas consecuencias si se tiene en cuenta que no se debe tomar en consideración la individualidad o constitución psíquica del autor, ya que tales cuestiones corresponden a la categoría de la culpabilidad si se admite que las mismas ya debieran ser relevantes en sede de tipicidad subjetiva.

Lo anterior permitiría encontrar fuertes puntos de apoyo a la decisión de los tribunales a la hora de no atribuir un comportamiento doloso a ciertos autores que incurran en un error de tipo psíquicamente condicionado, especialmente si se defiende un concepto de injusto personal que no pueda constituirse en caso 
de situaciones en que no se cumplan las exigencias emanadas de la evitabilidad individual como categoría central de la imputación subjetiva (García, 2012, p. 486). Obviamente, el desafío que se presenta es identificar aquellos casos en los que, efectivamente, se pueda sostener sin dudas que el autor no estaba en condiciones personales de alcanzar los conocimientos exigidos por el respectivo tipo subjetivo.

No obstante, y como ya se advirtió, un problema distinto es asumir que, en tales casos, en que no se satisfagan los requisitos del injusto personal y que, por ende, no se pueda aplicar una pena debido a que el comportamiento en particular no contraviene el ordenamiento jurídico, se desprenda, además, que no puede aplicarse una medida de seguridad.

\section{LA IMPOSICIÓN DE MEDIDAS DE SEGURIDAD PESE AL RECONOCIMIENTO DE QUE EN ESTOS CASOS NO SE ACTÚA CON DOLO}

Por otra parte, aun entendiendo que la ausencia de dolo impide satisfacer los requisitos de la tipicidad $\mathrm{y}$, por tanto, del injusto, esto no supone necesariamente que se deba negar, sin más, la procedencia de medidas de seguridad, ya que tal posibilidad también dependerá de la respuesta al problema de las condiciones que permiten dictar una medida de seguridad y, específicamente, al del significado que se le deba otorgar a la exigencia previa de un comportamiento típico y antijurídico. Molina ha mostrado adecuadamente la forma en que se ha expresado este problema por parte de la doctrina penal:

\begin{abstract}
Se trata de la calificación de ciertos hechos en los que sin duda falta algún elemento subjetivo de los que la teoría considera imprescindibles para la presencia de una acción típica - hay una causa de ausencia de acción, un error de tipo o falta un elemento subjetivo del injusto-, y en los que, sin embargo, intuitivamente percibimos que deberían aplicarse las consecuencias jurídicas ligadas al injusto penal, y muy especialmente una medida de seguridad. (2008, p. 115).
\end{abstract}

En este sentido, se ha señalado que, aun asumiendo que en estos casos no se pueda afirmar que el sujeto actúe con dolo, se podría negar que de lo anterior se derive necesariamente la conclusión a la que arriba cierto sector de la doctrina, es decir, la improcedencia de las medidas de seguridad.

Así, por ejemplo, Silva (2003), sostiene que la anterior conclusión supone, en primer lugar, asumir que la imposición de una medida de seguridad a un inimputable requiere que "éste hubiera realizado, en el caso de un delito doloso, tanto el tipo objetivo como subjetivo" (p. 123), lo que no se cumpliría por faltarle el aspecto subjetivo del hecho respectivo. Con todo, tal punto de partida presupondría que "la concurrencia de un hecho objetiva y subjetivamente típico y no justificado constituye el presupuesto dogmático ineludible" tanto de la imposición de la pena como de la medida de seguridad (Silva, 2003, p. 124). Pero, por ello, la respuesta podría cambiar si no se parte de la premisa indicada y se pone en duda la corrección 
de los presupuestos de la imposición de medidas de seguridad de la que parte.

En lo que sí existe acuerdo es en que las medidas de seguridad solo pueden imponerse con posterioridad a la comisión de un hecho antijurídico para los casos en que se verifique peligrosidad, en sentido de probabilidad de comisión de un nuevo hecho antijurídico (Silva, 2003, p. 126). Lo que no parece obvio, según el mismo autor, es que el "significado de la exigencia de antijuridicidad" sea el mismo ya sea para la aplicación de la pena ya sea para la medida de seguridad. En este sentido ha señalado que:

\begin{abstract}
Cada vez resulta más difícil sostener que dicho concepto dominante de injusto, esto es, el de la doctrina del injusto personal, además de constituir —una vez que se le añada el juicio de culpabilidad- el presupuesto de la imposición de la pena al autor, constituya el denominador común de la accesoriedad de la participación, la agresión que puede dar lugar a la legítima defensa, el injusto al que se refiere el conocimiento de la antijuridicidad (...) y el que es propio del hecho desencadenante de la imposición de medidas de seguridad. (Silva, 2014, p. 8).
\end{abstract}

Esto quiere decir que, inclusive reconociendo que en los supuestos abordados se deba negar el comportamiento doloso, se podrían aplicar medidas de seguridad si se considera que la antijuridicidad a la que se refiere el legislador, para tales efectos, presenta un "carácter puramente objetivo, en el sentido de determinarse por la lesión objetivamente típica y no justificada de un jurídico-penal, sin que concurra elemento subjetivo alguno" (Silva, 2003, p. 126).

Para reforzar tal postura recurre a una serie de argumentos de índole teleológica relativos a la distinción entre la finalidad asignada a las penas y a las medidas de seguridad. Por tanto, para la aplicación de las medidas de seguridad debiera bastar, exclusivamente "el juicio de pasado en virtud del que se fija la conexión causal entre la enfermedad y la producción de una lesión típica y no justificada del bien jurídico-penal y el juicio de futuro (de pronóstico), según el cual cabe prever que eso pueda repetirse" (Silva, 2003, p. 128).

Tal posición pone de relieve un primer grupo de dificultades asociadas a la alegación de que frente a ciertas situaciones de alteración mental que imposibiliten que un sujeto conozca que realiza los elementos de un tipo penal, se extraiga como consecuencia la ausencia de dolo y, luego, la imposibilidad de aplicar medidas de seguridad por no cumplirse la exigencia de configuración de un hecho típico y antijurídico. Este planteamiento parece razonable en la medida en que parte de un presupuesto del todo correcto. La razón por la cual parece adecuada la noción de injusto personal que dice relación a la convicción de que solo puede ser considerado como un hecho injusto aquello que podría ser evitado a través del seguimiento de normas, por ende, la remisión a cierta capacidad del agente es imprescindible. 
Con todo, tal exigencia que tiene todo el sentido desde la perspectiva de la imposición de una pena, no necesariamente lo tiene para la imposición de una medida de seguridad. Por otra parte, y según la postura analizada, se debiera tener en cuenta que la exigencia de la constatación de la comisión de un hecho típico y antijurídico, desde la lógica de la imposición de las medidas de seguridad, está más bien dirigida, por una parte, a evitar la imposición de medidas predelictuales y, por otra, a evitar aplicar medidas de seguridad para casos en los que inimputables lleven a cabo una lesión típica, pero justificada del bien jurídico-penal. Como ha quedado de manifiesto, la solución de Silva pasa por examinar profundamente la corrección de la pretensión de equivalencia del concepto de "hecho típico y antijurídico" tanto para la aplicación de una pena a la de una medida de seguridad.

\section{CONCLUSIONES}

Frente al problema planteado al comienzo de este artículo, se debe concluir que resulta razonable sostener que, en determinadas situaciones en que un sujeto presenta ciertos trastornos mentales que no le permitan alcanzar el conocimiento requerido por el tipo penal, no podría atribuirse un comportamiento doloso al mismo.

Como se pudo apreciar, parte de la práctica judicial reconoce que el juicio de adscripción del dolo, como atribución de conocimiento, se hace tomando en cuenta las circunstancias personales del agente, incluyendo en estas la individualidad del sujeto. Así las cosas, pareciera que en el proceso conocido como de "imputación subjetiva" no bastaría el ponerse en lugar del autor sin considerar su capacidad para conocer el significado de su comportamiento, y que este realiza los elementos de un tipo penal en particular.

Por lo mismo, existe respaldo para afirmar que en tal circunstancia el sujeto actuaría en error de tipo —la mayoría de las veces invencible, debido a su condición psíquica-y que, por ello, se debe excluir el dolo, por ausencia de conocimiento. La respuesta al problema planteado, en definitiva, dependerá de los criterios o parámetros normativos a los que se adhiera para el juicio de adscripción del conocimiento y, por tanto, será muy distinta si se aboga por la inclusión de criterios individualizadores a criterios que no tomen en cuenta la individualidad del autor debido a que este sería un problema exclusivo de la categoría de la culpabilidad.

Como se señaló, este problema se presenta solo para el caso en que se adhiera a un concepto personal de injusto y no se entienda el dolo como una forma de culpabilidad. Al respecto, se señaló que la tendencia mayoritaria en nuestra doctrina es asumir este punto de partida como correcto y, por tanto, a incluir el dolo o la imprudencia en el tipo penal — como tipo subjetivo-y, por tanto, la ausencia de dolo excluye a su vez el comportamiento doloso, impidiendo que se satisfagan todos los requisitos del tipo penal. Es importante indicar que el problema del tratamiento del "error psíquicamente condicionado" no ha sido, aún, abordado por 
nuestra doctrina en forma acabada y que, incluso, tampoco se ha tratado en detalle el problema vinculado a la consideración de circunstancias personales - como puede ser la presencia de determinados trastornos mentales - para la imputación del dolo. Además, nuestros tribunales tienden a asumir que tales problemas son parte de la culpabilidad, sin indicar las razones por las cuales puede prescindirse de su análisis para la conformación del injusto personal.

Por otra parte, se pudo constatar que, debido a lo anteriormente expuesto, los tribunales, frente a la acreditación de enajenación mental por parte de un sujeto, y la verificación de su peligrosidad, tienden a dictar medidas de seguridad sin cuestionarse, en forma previa, si se cumplen los requisitos impuestos por el artículo 455 del Código Procesal Penal chileno, específicamente en lo que respecta a la tipicidad de la conducta. Más bien tienden a repetir fórmulas mecánicas de reconocimiento de que se está frente a un hecho típico y antijurídico. Sin embargo, se pudo analizar un fallo reciente de la Corte de Apelaciones de Valparaíso en el cual se rechaza la imposición de una medida de seguridad asumiendo que el error en el que actúa el acusado - condicionado absolutamente por su trastorno mental- excluía el dolo y, con ello, el injusto.

Por último, se debe advertir que, frente a tal conclusión, esto es, la imposibilidad de atribuir conocimiento al autor del delito en los casos en que el trastorno mental impida al sujeto formarse el conocimiento exigido por el tipo subjetivo y, por tanto, no pueda conformarse el injusto personal, se podría optar por dos vías diversas en lo que respecta a las consecuencias de tal declaración frente a la imposición de medidas de seguridad. Por una parte, el asumir que la exigencia de constatación de un hecho típico y antijurídico del artículo 455 del Código Procesal Penal chileno debe ser interpretada en términos equivalentes para el de la imposición de una pena - por tanto, como injusto personal-, lo que impediría que los tribunales aplicaran una medida de seguridad a quien actúa bajo tales circunstancias, o la de sostener que, en realidad, se trata de un concepto de injusto diverso, meramente objetivo, como ha indicado cierta doctrina. El fundamento de tal distinción vendría dado por la diversa finalidad que se le asigna a la pena frente a la finalidad propia de las medidas de seguridad, para las cuales no tendría sentido la exigencia de constatar en forma previa el acaecimiento de un hecho entendido como un "injusto personal", sino que bastaría, más bien, un hecho lesivo para bienes jurídico-penalmente relevantes. Todo esto, con el propósito de evitar la imposición de medidas de seguridad predelictuales.

\section{REFERENCIAS}

Cillero, M. (2011). Artículo 10. En H. Hernández y J. Couso (Eds.), Código Penal Comentado. Parte general (pp.176-200). Santiago de Chile: Legal Publishing Chile.

Cury, E. (2005). Derecho Penal. Parte general (7. ${ }^{\text {a Ed.). }}$ Santiago de Chile: Ediciones Universidad Católica de Chile. 
Etcheberry, A. (1998). Derecho Penal. Parte general (3. ${ }^{\text {a }}$ Ed.). Santiago de Chile: Editorial Jurídica de Chile.

Fakhouri, Y. (2009). Teoría del dolo vs. teoría de la culpabilidad: un modelo para afrontar la problemática del error en Derecho penal. InDret, (4), 1-29. Recuperado de http://www. indret.com/pdf/671.pdf

García, P. (2012). Derecho Penal. Parte general (2. Ed.). Lima: Jurista Editores.

Garrido, M. (2003). Derecho Penal. Parte general

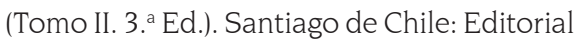
Jurídica de Chile.

Hernández, H. (2011). Artículo 1. En H. Hernández Basualto y J. Couso Salas (Eds.), Código Penal comentado. Parte general. Santiago de Chile: Legal Publishing Chile.

Hruschka, J. (2005). Imputación y Derecho Penal: estudios sobre la teoría de la imputación. Navarra: Thomson Aranzadi.

Joshi, U. (1989). Algunas consecuencias que la inimputabilidad puede tener para la antijuricidad. Anuario de Derecho Penal y Ciencias Penales, 42 (1), 125-140.

Kindhäuser, U. (2008). El tipo subjetivo en la construcción del delito. InDret, (4), 1-35. Recuperado de https://www.raco.cat/index. php/InDret/article/view/124309/172282

Kindhäuser, U. (2006). Imputación objetiva y subjetiva en el delito doloso. Anuario de Derecho Penal y Ciencias Penales, 59(1), 63-81.

Luzón, D. (2016). Derecho Penal. Parte general (3. Ed.). Buenos Aires: Editorial B de F.

Mañalich, J. (2005). Condiciones generales de la punibilidad. Rev. D. UAI, (2), 387-481.

Molina, F. (2008). Error de tipo derivado de anomalías o alteraciones psíquicas: un difícil desafío para la teoría del delito. Revista Cuatrimestral de las Facultades de Derecho y Ciencias Económicas y Empresariales, (74), 113-144.
Morse, S. y Hoffman, M. (2007). The Uneasy Entende between Legal Insanity and Mens Rea: Beyond Clark v. Arizona. Journal of Criminal Law and Criminology, 97(4), 1071-1150.

Novoa, E. (2005). Curso de Derecho Penal chileno. Parte

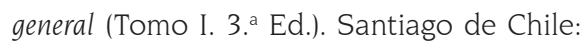
Editorial Jurídica de Chile.

Paredes, J., Díaz y García, M. y De Vicente, J. (2001). Argumentación y prueba en la determinación del dolo (comentario a la STS 24-10-1989, Pte.: García Ancos). Revista de Derecho Penal y Criminología, 2(8), 329-353.

Politoff, S., Matus, J. y Ramírez, M. (2003). Lecciones

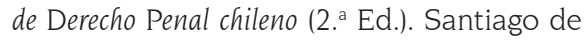
Chile: Editorial Jurídica de Chile.

Ragués, R. (2002). El dolo y su prueba en el proceso penal. Barcelona: José María Bosch Editor.

Ragués, R. (2004). Consideraciones sobre la prueba del dolo. Revista de Estudios de la Justicia, (4), 13-26.

Sánchez-Málaga, A. (2015). El dolo: ¿fenómeno espiritual o atribución normativa? Themis. Revista de Derecho, (68), 61-75.

Silva, J. (2003). Normas y acciones en Derecho Penal. Buenos Aires: Hammurabi.

Silva, J. (2014). ¿Adiós a un concepto unitario de injusto en la teoría del delito? A la vez, una breve contribución a la teoría de las medidas de seguridad. InDret, (3), 1-20.

Zaffaroni, E., Alagia, A. y Slokar, A. (2002). Derecho Penal. Parte general. (2. ${ }^{a}$ Ed.). Buenos Aires: Editorial Ediar. 